\section{Endobronchial Optical Coherence Tomography for Low-Risk Microscopic Assessment and Diagnosis of Idiopathic Pulmonary Fibrosis In Vivo}

\section{To the Editor:}

Diagnostic certainty of idiopathic pulmonary fibrosis (IPF) is critical to determine prognosis and therapeutic options. The U.S. Food and Drug Administration-approved antifibrotic agents pirfenidone and nintedanib are currently only indicated for IPF, and immunosuppressants frequently used to treat other interstitial lung diseases (ILD) have been associated with worse outcomes when used for IPF (1-4). According to current guidelines, a diagnosis of IPF requires identification of a usual interstitial pneumonitis (UIP) pattern by high-resolution computed tomography (HRCT) and/or microscopic tissue examination (1). The diagnosis can be made by HRCT alone in $\sim 50 \%$ of cases, when all UIP criteria are met, including honeycombing (1). However, the inability to visualize microscopic honeycombing $(<2-3 \mathrm{~mm})$ or distinguish honeycomb from traction bronchiectasis is a frequent cause of diagnostic uncertainty $(5,6)$. When HRCT is inconclusive, surgery is required to obtain wedge biopsies for microscopic evaluation (1), which is associated with morbidity/mortality risks in patients with IPF (7-9). This mandates a cautious approach to employing surgical biopsy for diagnosis, and precludes its repeated use to assess disease progression.

We demonstrate the use of in vivo endobronchial optical coherence tomography (OCT) as a minimally invasive method to microscopically assess and diagnose UIP/IPF without tissue removal. OCT provides rapid, three-dimensional imaging with microscopic resolution $(<10 \mu \mathrm{m})$ well beyond HRCT capabilities, and can assess significantly larger tissue volumes $(\sim 100 \times)$ than can be assessed microscopically from surgical biopsies (10). OCT is performed by passing a narrow catheter through a standard bronchoscope working channel out to the peripheral lung and conducting helical scanning with pullback lengths up to $10 \mathrm{~cm}$. Some of the results of these studies have been previously reported in the form of an abstract (11-13).

Supported in part by NIH grants K23HL132120, T32CA009216, and R01CA167827.

Author Contributions: L.P.H. contributed to the study design; collection, analysis, and interpretation of data; and drafting and critical review of the manuscript; and read and approved the final version. D.C.A., J.C.W. M.L., A.M., A.S., T.V.C., M.M.-K., E.J.M., and R.L.K. contributed to the collection and analysis of data, and critical review of the manuscript, and read and approved the final version. H.G. contributed to the collection of data and critical review of the manuscript, and read and approved the final version. A.M.T. contributed to the study design, analysis and interpretation of data, and drafting and critical review of the manuscript, and read and approved the final version. M.J.S. contributed to the study design, analysis and interpretation of data, and drafting and critical review of the manuscript; read and approved the final version; had full access to all of the data in the study; and had final responsibility for the decision to submit for publication.

Originally Published in Press as DOI: 10.1164/rccm.201707-1446LE on September 21, 2017

\section{Methods}

We conducted a pilot study in five sequential patients with ILD undergoing video-assisted thoracic surgical biopsy for diagnosis at Massachusetts General Hospital. Endobronchial OCT was performed before each biopsy. Imaging locations were selected based on sites of abnormalities identified on HRCT, with four to six OCT pullbacks obtained per patient. The total time needed to collect all OCT data was $<6$ minutes. OCT imaging was interpreted independently of biopsy. The OCT images in Figures 1 and 2 are representative still frames selected from larger volumetric datasets. Patients consented to participate in the study (approved by the Partners Human Research Institutional Review Board, \#2015-P001345). A summary of the cases is presented in Table 1.

\section{Results}

Endobronchial OCT accurately identified UIP/IPF by detecting salient microscopic honeycombing and other UIP features in patients with nondiagnostic HRCT. In cases 1-3, nondiagnostic HRCT showed bibasilar, subpleural reticular opacities but no honeycombing. OCT showed microscopic features of UIP (Figure 1A), including multifocal microscopic honeycombing seen as irregular, cystic, signal-void spaces embedded in peripheral, destructive, signal-intense fibrosis with loss of alveolar architecture, just beyond the bronchiolar epithelium. OCT visualized spatial heterogeneity as regions of preserved alveoli adjacent to regions of fibrosis. Subsequent biopsies (Figure 1B) independently confirmed the UIP diagnosis in all patients.

Endobronchial OCT accurately differentiated traction bronchiectasis from microscopic honeycombing to identify non-UIP/IPF ILD in a patient with a false-positive diagnosis of UIP/IPF by HRCT. In case 4, HRCT showed subpleural reticular opacities, traction bronchiectasis, and honeycomb change, consistent with "definitive UIP" based on current guidelines (1). Because the patient had a history of juvenile arthritis and may have had an autoimmune-related ILD, a surgical biopsy was obtained. OCT (Figure 1C) visualized numerous foci of traction bronchiectasis as cystic airway branchpoints connected to the main airway, with nondestructive interstitial fibrosis in alveolar walls. No honeycombing or other UIP features were seen. A subsequent biopsy (Figure 1D) independently confirmed all of the features visualized on OCT, including traction bronchiectasis and interstitial fibrosis with a nonspecific interstitial pneumonitis-like pattern, which led to a non-UIP/IPF ILD diagnosis. Upon retrospective assessment at a multidisciplinary conference, it was determined that the honeycombing seen on HRCT was a false positive due to traction bronchiectasis.

Endobronchial OCT identified microscopic honeycombing and other UIP features in a patient with nondiagnostic HRCT and indeterminate surgical biopsy. In case 5, HRCT showed bibasilar subpleural reticular opacities, with mild ground-glass opacities and traction bronchiectasis, but no honeycombing. OCT (Figure 2A) showed dense, destructive subpleural fibrosis, with loss of alveolar architecture. Foci of irregular, cystic, multilayered, signal-void spaces were embedded within fibrosis, 

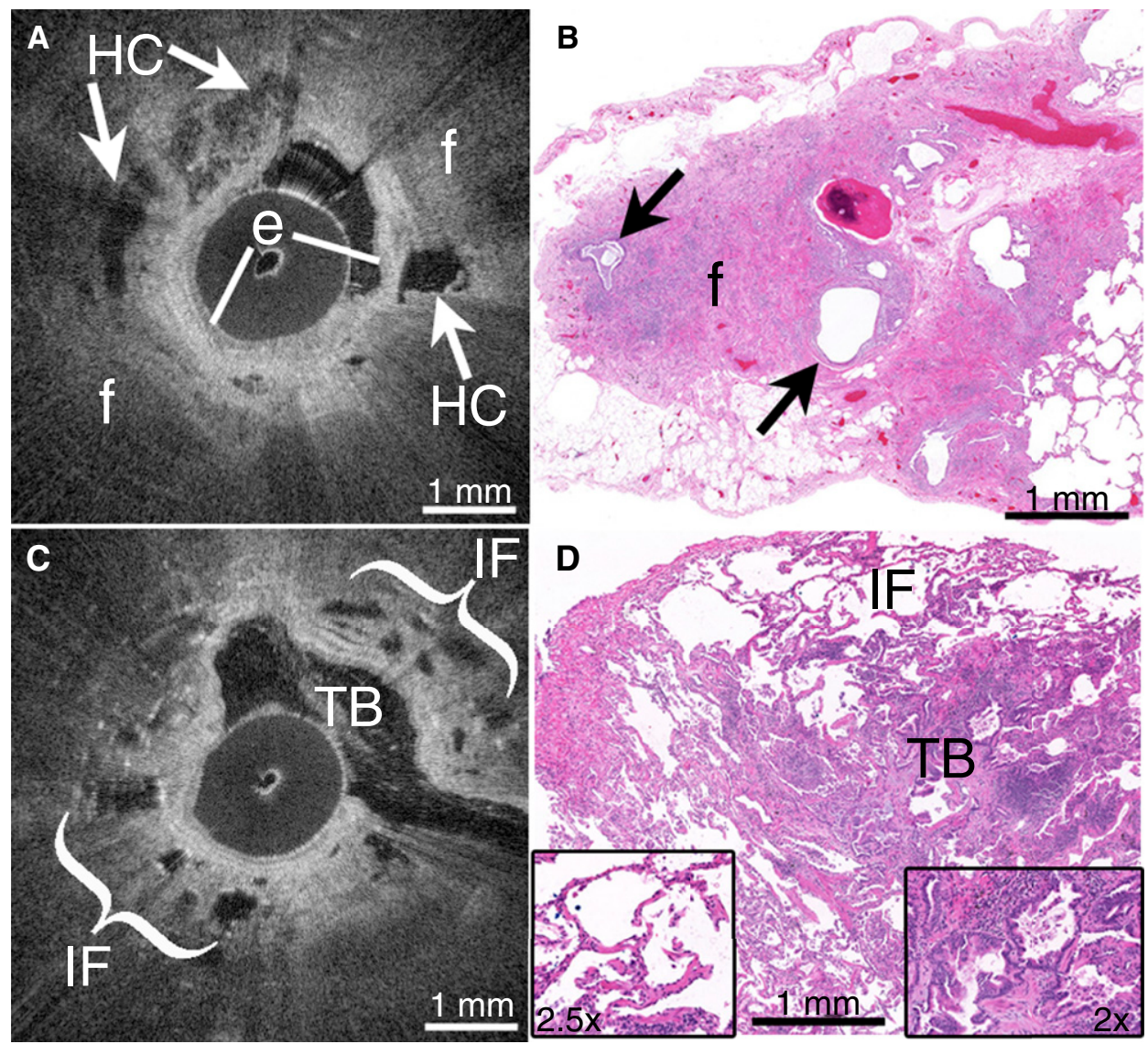

Figure 1. (A) In vivo endobronchial optical coherence tomography (OCT) accurately identified features of usual interstitial pneumonitis (UIP)/idiopathic pulmonary fibrosis (IPF), including multifocal microscopic honeycombing (HC, arrows) as layered cystic structures embedded within peripheral, destructive fibrosis (f) beyond the bronchiolar epithelium (e). (B) Subsequent surgical lung biopsies were independently diagnosed as UIP, and confirmed the presence of peripheral fibrosis (f) and microscopic honeycombing (arrows). (C) OCT accurately identified traction bronchiectasis (TB) as cystic airway branchpoints, and nondestructive interstitial fibrosis (IF) in alveolar walls in non-UIP/IPF interstitial lung disease (ILD). (D) Subsequent surgical lung biopsy independently confirmed all features visualized on OCT, including traction bronchiectasis (TB, right inset) and interstitial fibrosis (IF) in alveolar walls (left inset). No honeycombing or other UIP features were seen, and the biopsy was diagnosed as a non-UIP/IPF ILD.
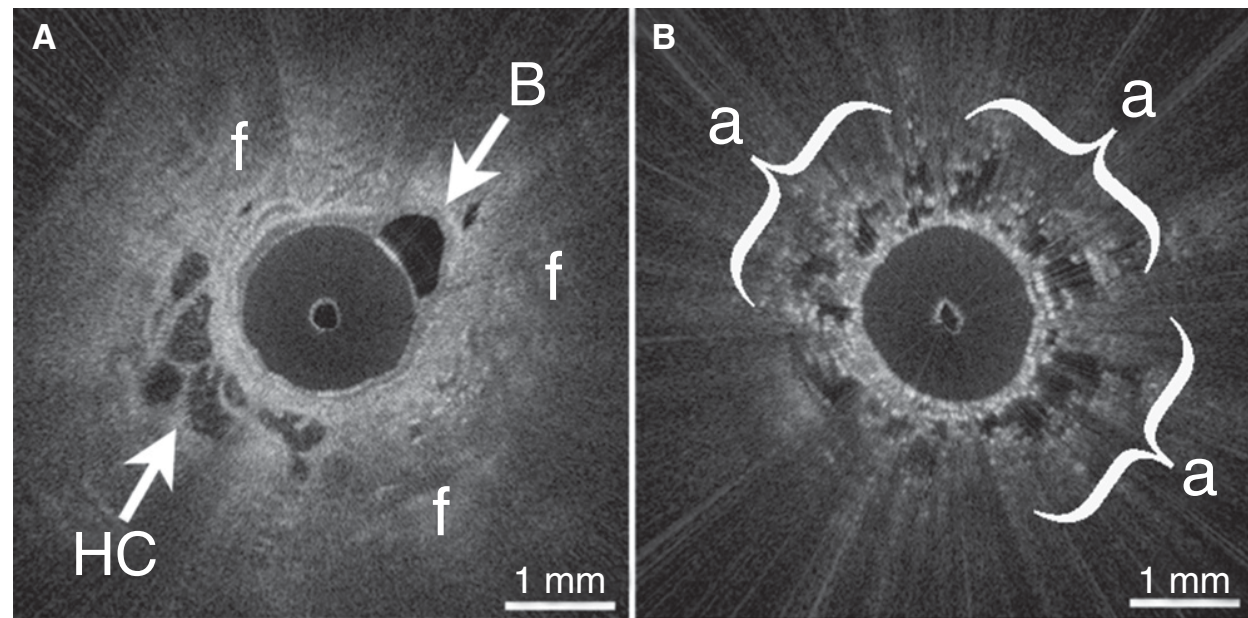

Figure 2. (A) In vivo endobronchial optical coherence tomography (OCT) identified microscopic honeycombing (HC) and destructive peripheral fibrosis ( $f$ ) in a patient with nondiagnostic high-resolution computed tomography and indeterminate lung biopsy. A branching bronchiole (B) is also visible. (B) OCT visualized spatial heterogeneity as regions of preserved alveoli (a) adjacent to fibrosis. 
Table 1. Summary of Patients with Interstitial Lung Disease Who Underwent In Vivo Endobronchial Optical Coherence Tomography

\begin{tabular}{lcccc}
\hline Case No. & Age/Sex & HRCT Interpretation & $\begin{array}{c}\text { In Vivo Endobronchial } \\
\text { OCT Diagnosis }\end{array}$ & Surgical Lung \\
& & & UIP & UIP \\
1 & $56 / \mathrm{M}$ & Nondiagnostic & UIP \\
2 & $74 / \mathrm{M}$ & Nondiagnostic & UIP \\
3 & $69 / \mathrm{M}$ & Nondiagnostic & UIP & Non-UIP/IPF ILD \\
5 & $52 / \mathrm{F}$ & UIP & UIP & Indeterminate \\
5 & $64 / \mathrm{M}$ & Nondiagnostic & ILD & \\
\hline
\end{tabular}

Definition of abbreviations: HRCT = high-resolution computed tomography; ILD = interstitial lung disease; IPF = idiopathic pulmonary fibrosis; OCT = optical coherence tomography; UIP = usual interstitial pneumonitis.

${ }^{*}$ Reviewed by five pathologists: interpreted by three as UIP without clear honeycomb; by one as indeterminate, UIP possible; and by one as chronic organizing pneumonia.

consistent with honeycombing. Regions of preserved alveolar architecture (Figure 2B) were seen adjacent to areas of destructive fibrosis. A subsequent lung biopsy showed dense, destructive subpleural fibrosis, and infrequent individual cystic structures. Owing to the difficulty of interpreting the biopsy, five pathologists with ILD expertise reviewed the slides. Three of five pathologists stated that the biopsy was consistent with UIP but without definitive honeycombing. One said that UIP was possible but the biopsy was insufficient for a definitive diagnosis. All four pathologists postulated that the infrequent cystic structures could be honeycombing. The fifth pathologist stated that the biopsy was consistent with chronic organizing pneumonia, and was not UIP. Although the majority agreed that the biopsy looked like UIP, the lack of clear honeycombing precluded a definitive diagnosis.

\section{Discussion}

With total imaging times of $<6$ minutes, a fraction of the time required for surgical biopsy, we have shown that minimally invasive endobronchial OCT can establish accurate diagnoses of UIP/IPF and non-UIP/IPF ILD in patients with nondiagnostic HRCT or with a false-positive diagnosis of UIP/IPF by HRCT. OCT can detect and distinguish microscopic features of UIP/IPF and non-UIP/IPF ILD, including fibrosis extent and distribution, microscopic honeycombing $(<2 \mathrm{~mm})$ not visualized by HRCT, and traction bronchiectasis confused with honeycombing on HRCT. Fibroblastic foci, a histologic criterion for UIP/IPF, are not visible by HRCT and thus are not part of the HRCT criteria for UIP/IPF (1). The distinction between definitive and probable UIP/IPF diagnoses by HRCT rests on the detection of honeycombing. Therefore, we anticipate that accurate recognition of honeycombing by OCT, rather than assessment of fibroblastic foci, will provide high diagnostic accuracy, as demonstrated by the presented cases.

UIP/IPF is characterized by patchy disease with spatial heterogeneity. Thus, it is well known that lung biopsy can be an imperfect diagnostic technique owing to small tissue volumes and sampling error. Endobronchial OCT images significantly larger tissue volumes and can access more distinct locations than biopsies, and thus is less likely to suffer from sampling errors. It is postulated that in case 5, OCT may have visualized honeycomb foci in regions that were not captured within the more limited surgical biopsy tissue.

The above case vignettes support the potential of OCT to serve as a low-risk microscopic complement to HRCT for the assessment and diagnosis of UIP/IPF in vivo without surgery or tissue resection. Further validation studies are needed to assess the sensitivity/specificity of OCT for UIP/IPF diagnosis. OCT, by virtue of its minimal invasiveness, can be performed under conscious sedation and allows patients to be studied repeatedly over time. This will provide unique opportunities to investigate the natural history of IPF pathogenesis and assess the efficacy of new therapeutic agents longitudinally at the microscopic level, which would be a tremendous tool in both clinical and research settings.

Author disclosures are available with the text of this letter at www.atsjournals.org.

Lida P. Hariri, M.D., Ph.D.

David C. Adams, Ph.D.

John C. Wain, M.D.

Michael Lanuti, M.D.

Ashok Muniappan, M.D.

Amita Sharma, M.D.

Massachusetts General Hospital

Boston, Massachusetts

and

Harvard Medical School

Boston, Massachusetts

Thomas V. Colby, M.D.

Mayo Clinic

Scottsdale, Arizona

Mari Mino-Kenudson, M.D.

Eugene J. Mark, M.D.

Richard L. Kradin, M.D.

Massachusetts General Hospital

Boston, Massachusetts

and

Harvard Medical School

Boston, Massachusetts 
Hannah Goulart, B.S.

Massachusetts General Hospital

Boston, Massachusetts

Andrew M. Tager, M.D.

Melissa J. Suter, Ph.D.

Massachusetts General Hospital

Boston, Massachusetts

and

Harvard Medical School

Boston, Massachusetts

\section{References}

1. Raghu G, Collard HR, Egan JJ, Martinez FJ, Behr J, Brown KK, et al.; ATS/ERS/JRS/ALAT Committee on Idiopathic Pulmonary Fibrosis. An official ATS/ERS/JRS/ALAT statement: idiopathic pulmonary fibrosis: evidence-based guidelines for diagnosis and management. Am J Respir Crit Care Med 2011;183: 788-824.

2. Raghu G, Anstrom KJ, King TE Jr, Lasky JA, Martinez FJ; Idiopathic Pulmonary Fibrosis Clinical Research Network. Prednisone, azathioprine, and $\mathrm{N}$-acetylcysteine for pulmonary fibrosis. $\mathrm{N}$ Engl $J$ Med 2012;366:1968-1977.

3. Richeldi L, du Bois RM, Raghu G, Azuma A, Brown KK, Costabel U, et al.; INPULSIS Trial Investigators. Efficacy and safety of nintedanib in idiopathic pulmonary fibrosis. N Engl J Med 2014;370: 2071-2082.

4. King TE Jr, Bradford WZ, Castro-Bernardini S, Fagan EA, Glaspole I, Glassberg MK, et al.; ASCEND Study Group. A phase 3 trial of pirfenidone in patients with idiopathic pulmonary fibrosis. $N$ Engl $J$ Med 2014;370:2083-2092.

5. Gotway MB, Freemer MM, King TE Jr. Challenges in pulmonary fibrosis. 1: Use of high resolution CT scanning of the lung for the evaluation of patients with idiopathic interstitial pneumonias. Thorax 2007;62: 546-553.

6. Watadani T, Sakai F, Johkoh T, Noma S, Akira M, Fujimoto K, et al. Interobserver variability in the CT assessment of honeycombing in the lungs. Radiology 2013;266:936-944.

7. Utz JP, Ryu JH, Douglas WW, Hartman TE, Tazelaar HD, Myers JL, High short-term mortality following lung biopsy for usual interstitial pneumonia. Eur Respir J 2001;17: 175-179.

8. Fibla JJ, Brunelli A, Cassivi SD, Deschamps C. Aggregate risk score for predicting mortality after surgical biopsy for interstitial lung disease. Interact Cardiovasc Thorac Surg 2012;15:276279.

9. Kreider ME, Hansen-Flaschen J, Ahmad NN, Rossman MD, Kaiser LR, Kucharczuk JC, et al. Complications of video-assisted thoracoscopic lung biopsy in patients with interstitial lung disease. Ann Thorac Surg 2007;83:1140-1144.

10. Yun SH, Tearney GJ, Vakoc BJ, Shishkov M, Oh WY, Desjardins AE, et al. Comprehensive volumetric optical microscopy in vivo. Nat Med 2006;12:1429-1433.

11. Hariri LP, Adams DC, Colby TV, Tager AM, Suter MJ. Assessing idiopathic pulmonary fibrosis (IPF) with bronchoscopic OCT (conference presentation). Proc SPIE 2016;9697:969710.

12. Hariri LP, Adams DC, Lanuti M, Wain JC, Tager AM, Suter MJ. Bronchoscopic optical coherence tomography as a low risk method for microscopic diagnosis of idiopathic pulmonary fibrosis. Am J Respir Crit Care Med 2016;193: A2608.

13. Hariri LP, Adams DC, Lanuti M, Wain JC, Muniappan A, Goulart H, et al. In vivo endobronchial optical imaging for microscopic assessment and diagnosis of idiopathic pulmonary fibrosis. Am J Respir Crit Care Med 2017;195:A6782.

Copyright (C) 2018 by the American Thoracic Society

\section{The Y Chromosome Plays a Protective Role in Experimental Hypoxic Pulmonary Hypertension}

\author{
To the Editor:
}

Pulmonary arterial hypertension (PAH) is a chronic pulmonary vascular disease characterized by elevated pulmonary arterial pressure and vascular remodeling leading to right ventricular (RV) failure and death (1). $\mathrm{PAH}$ in general, and its idiopathic form in particular, has long been considered to be a disease of young women, with female sex itself being a risk factor (1). Surprisingly, being female is protective against the development of pulmonary hypertension $(\mathrm{PH})$ in various animal models (2). Because this effect has largely been attributed to the protective effects of estradiol, the phenomenon is known as the "estrogen paradox" $(3,4)$. One way to resolve this paradox is to consider that sex hormones alone are not sufficient to explain the marked sex bias observed in $\mathrm{PH}$ in humans.

Here, we report that the type of sex chromosome (XX vs. XY) contributes to sex differences in $\mathrm{PH}$ mouse models. Sex chromosomes and sex hormones are both known to act directly on tissues to produce sex differences in physiology and disease (5). Mouse studies in which the number and type of sex chromosomes were independent of gonadal sex indicated that sex chromosomes contribute to sex differences in autoimmune disease, metabolic dysfunction, neurodegeneration, and cardiovascular disease $(6,7)$. Therefore, genes encoded on the sex chromosomes have already been shown to have strong effects that contribute to sex differences in various phenotypes, including disease susceptibility and progression. We investigated, for the first time, the role of sex chromosomes (in the absence of gonadal hormones) in the development of hypoxia-induced $\mathrm{PH}$, using the informative "four core genotypes" (FCG) and XY* mouse models.

The FCG model produces mice in which sex chromosomes are independent of gonadal sex (XX and XY mice with testes [males], and $\mathrm{XX}$ and XY mice with ovaries [females]), allowing the effects of sex chromosomes to be assessed independently of gonadal sex (5). To eliminate the effect of gonadal hormones in adulthood, FCG mice were gonadectomized (GDX) at 75 days after birth, and 30 days later were placed in hypoxia (10\% oxygen) or normoxia for 3 weeks. We found that hypoxic XY mice, irrespective of gonadal sex, developed less severe $\mathrm{PH}$ than XX mice. RV systolic pressures (RVSPs) were significantly lower in XY mice than in XX mice (RVSP: mean \pm SEM, $43.31 \pm$ $5.72 \mathrm{~mm} \mathrm{Hg}$ in XX females and $46.51 \pm 4.52$ in XX males vs. $33.17 \pm$ 2.36 in XY females and $40.4 \pm 2.31$ in XY males). Lower RVSPs in XY mice were accompanied by the presence of less severe pulmonary vascular remodeling (percent wall thickness: $23.84 \pm 3.14$ in XY females

Supported by an American Thoracic Society Proof of Concept grant (S.U.), American Heart Association grant 17PRE33420159 (C.M.C.), and NHH grants R01HL129051 (M.E.), R01HL131182 (M.E. and A.P.A.), and R01HD076125 (A.P.A.).

Author Contributions: Conception and design: S.U., C.M.C., Y.I., S.M., M.V., S.S., A.C., A.P.A., and M.E. Acquisition, analysis, or interpretation of data: S.U., C.M.C., Y.I., S.M., M.V., S.S., A.C., A.P.A., and M.E. Drafting the work or revising it critically for important intellectual content: S.U., C.M.C., Y.I., S.M., M.V., S.S., A.C., A.P.A., and M.E. Final approval of the version to be published: S.U., C.M.C., Y.I., S.M., M.V., S.S., A.C., A.P.A., and M.E. Agreement to be accountable for all aspects of the work in ensuring that questions related to the accuracy or integrity of any part of the work are appropriately investigated and resolved: S.U., C.M.C., Y.I., S.M., M.V., S.S., A.C., A.P.A., and M.E.

Originally Published in Press as DOI: 10.1164/rccm.201707-1345LE on September 21, 2017 